\title{
Assessment of Non-Verbal Communication Online Job Recruitment Using Gray Level Co-Occurrence Matrix and Fuzzy C-Means Algorithm
}

\author{
Anita Sindar Sinaga \\ Department of Informatics Engineering, STMIK Pelita Nusantara, Iskandar Muda 1 Medan 20154 Indonesia \\ *Corresponding author. Email: haito_ita@yahoo.com
}

\begin{abstract}
Communication contains two dimensions, verbal and non-verbal. Non-verbal communication behavior was evaluated using the feature extract calculation of the Gray Level Co-Occurrence Matrix (GLCM). Extraction formula consists of feature, contrast, energy, entropy and homogeneity at rotation 00, 450, 900 and 1350. The results of the GLCM extraction calculation are then classified using the Fuzzy C-Means algorithm. Fuzzy C-Means (FCM) is used in pattern recognition. Data identification on the FCM is determined based on the degree of membership which has a value between 0 and 1 . This study processes the applicant's offline video data. Identify expressions that indicate doubt, confusion and optimism. The assessment of the 10 videos was taken with the dominant optimistic expression extraction loop, it was obtained that the 7 th video had the highest percentage of optimistic facial expressions.
\end{abstract}

Keywords: Offline Video, Non-Verbal Communication, LBP, FCM, GLCM.

\section{INTRODUCTION}

Job fields that prioritize personnel who have to appear in front of people online recruitment opportunities provide many advantages. Focus more on body language to support biodata. Assessment of applicants through video recordings from Skype, offline videos. Online recruitment systems can be used to analyse nonverbal communication skills. Communication contains two dimensions, verbal and nonverbal. Nonverbal communication behaviours such as expressions of happiness, sadness and other facial expressions were evaluated using the Gray Level Co-Occurrence Matrix (GLCM) feature extract [1]. From the results of GLCM extraction, then classifying the recruitment using the Fuzzy C-Means Algorithm. Fuzzy C-Means (FCM) are used in pattern recognition [2]. The data grouping in the FCM is determined based on the degree of membership which has a value between 0 and 1 . A co-occurrence matrix is a matrix that is defined over an image to be the distribution of co-occurring value at a given offset [3]. The GLCM is a classic method of texture feature extraction, which is effective in image recognition, image segmentation, image retrieval, image classification, and texture analysis methods [4].
Extraction plays a basic role focusing on the feature extraction technique, while most of the recognition systems implementation is employed the binary representation in the feature extraction. Using cooccurrence matrix, feature vector can be extracted in several ways [5]. Gray Level Co-occurrence Matrix is a matrix that describes the frequency with which a pair of two pixels with a certain intensity in the distance (d) and orientation to the angle $(\theta)$ specified in the image [6]. The $\mathrm{K}-\mathrm{NN}$ using GLCM and wavelet decomposition texture feature performed very well on classifying acne with an accuracy of $100 \%$ [7]. This data will be processed by matching with normal expression data using template matching method so that can know where there is movement. In this section we present use of Fuzzy cmeans clustering algorithm [8].

\section{METHOD}

Representation of digital video information through a set of acquired images and displayed according to scanning system standards, frame rate, and frame size used by the video [9]. The research stages are shown in Figure 1. 


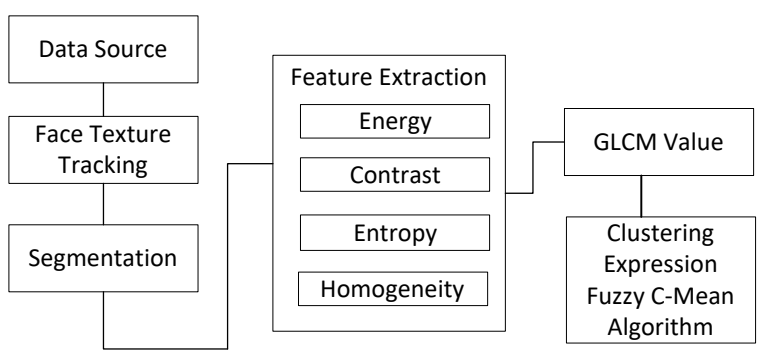

Figure 1 The research steps

The implementation stage of the GLCM begins with the value of the LBP calculation is obtained as input to the GLCM method. Normalization of a symmetrical matrix with the calculation of the distribution of Energy, Entropy, Contrast, Homogeneity [10]. The two-pixel neighbor directional angle is a combination of a single angle matrix and a composite multiple angle matrix having the image pixel value is $0^{\circ}, 45^{\circ}, 90^{\circ}$ and $135^{\circ}$ of GLCM method, Figure 2.

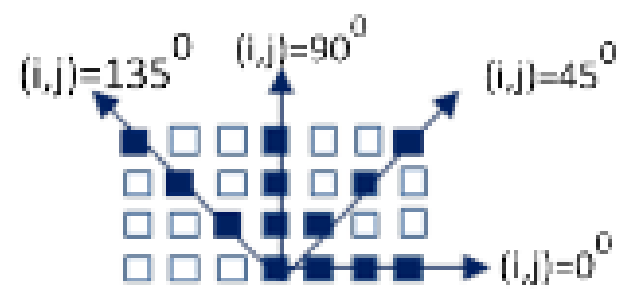

Figure 2 Co-ocurence angle GLCM method

Grouping of nonverbal communication expressions using Fuzzy Clustering Means. Clustering using FCM algorithm:

\section{Determine}

The matrix is $n \times m$ in size, where $n$ is the number of data to be clustered and $m$ is number of variables.

2. The form of the initial partition matrix $U$ (degree of membership in the cluster); The initial partition matrix is randomly generated.

3. Calculate the center of cluster $\mathrm{V}$ for each cluster.

4. Improve the degree of membership of each data in each cluster.

\begin{tabular}{|c|c|c|c|c|c|c|c|}
\hline 0 & 0 & 0 & 0 & 0 & 0 & 0 & 0 \\
\hline 23 & 17 & 1 & 0 & 0 & 0 & 0 & 0 \\
\hline 17 & 1114 & 151 & 0 & 0 & 0 & 0 & 0 \\
\hline 0 & 138 & 3580 & 291 & 2 & 0 & 0 & 0 \\
\hline 0 & 0 & 254 & 4826 & 223 & 13 & 0 & 0 \\
\hline 0 & 0 & 10 & 198 & 3301 & 105 & 3 & 0 \\
\hline 0 & 0 & 0 & 7 & 95 & 264 & 106 & 0 \\
\hline 0 & 0 & 0 & 0 & 3 & 63 & 1451 & 0 \\
\hline
\end{tabular}

\begin{tabular}{|c|c|c|c|c|c|c|c|}
\hline 0 & 0 & 0 & 0 & 0 & 0 & 0 & 0 \\
\hline 0 & 23 & 17 & 0 & 0 & 0 & 0 & 0 \\
\hline 0 & 17 & 1114 & 138 & 0 & 0 & 0 & 0 \\
\hline 0 & 1 & 151 & 3580 & 254 & 10 & 0 & 0 \\
\hline 0 & 0 & 0 & 291 & 4826 & 198 & 7 & 0 \\
\hline 0 & 0 & 0 & 2 & 223 & 3301 & 95 & 3 \\
\hline 0 & 0 & 0 & 0 & 13 & 105 & 264 & 63 \\
\hline 0 & 0 & 0 & 0 & 0 & 3 & 106 & 1451 \\
\hline
\end{tabular}

5. Determine the criteria for terminating the iteration, namely the change in the matrix in the current iteration and the previous iteration.

\section{RESULT AND DISCUSSION}

The study of body posture, movement, gesture, and facial expressions are called kinesics. This study focuses on eye, mouth and face movements. Offline video data is extracted into several frames according to duration. There are 10 videos that are processed starting from tracking, resulting in a set of frames. taken frames expressing Optimistic, Doubtful, Confused from eyes, mouth and face. Mean gray level shows the color graph of the video conversion into multiple frames, Figure 3.
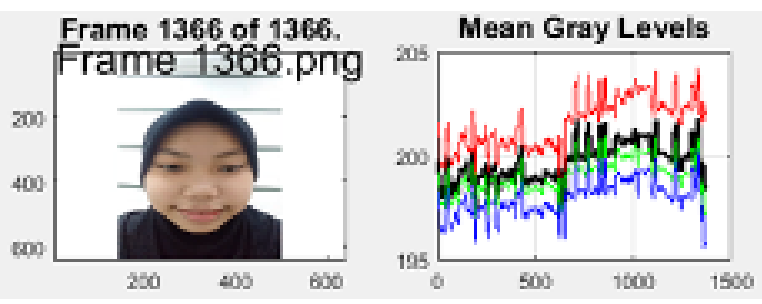

Figure 3 Convert video to frame by frame shows

\subsection{Results}

GLCM is used to identify objects with a statistical distribution followed by recognizing the pattern of rotation of the matrix at a specified degree. the assessed part of the face Non-Verbal Communication Expression, Figure 4.

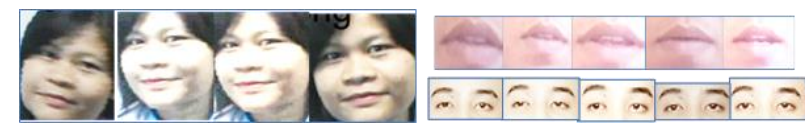

Figure 4 Non-verbal communication expression

After the co-occurrence matrix value is obtained, the next process is to calculate the statistical characteristic value. The statistical features to be calculated are, Table 1.

Figure 5 Co-occurrence matrix and transpose matrix 
Table 1. GLCM formula extraction

\begin{tabular}{|c|c|l|}
\hline Extraction & Formula & \multicolumn{1}{|c|}{ Explanation } \\
\hline Contras & $\sum_{i, j=0}^{n-1} p(i, j) \cdot(i-j)^{2}$ & Presents the degree of grayscale differences that appear in an image \\
\hline Energy & $\sum_{i, j=0}^{n-1} p(i, j)^{2}$ & $\begin{array}{l}\text { High energy when the pixel values are similar to each other, whereas } \\
\text { a small value indicates heterogeneous GLCM normalization. }\end{array}$ \\
\hline Entropy & $-\sum_{i, j=0}^{n-1} p(i, j) \log p(i-j)$ & A measure of the irregularity of the gray level in the image \\
\hline Comogeneity & $\sum_{i, j=0}^{n-1} p(i, j) /[1+(i-j)]^{2}$ & Grayscale of the adjacent 2 pixel resolution \\
\hline$\sum_{i} \sum_{j} \frac{(i-\mu i)(j-\mu j) p_{(i, j)}}{\sigma i \sigma j}$ & $\begin{array}{l}\text { Measure of the linear dependence between the gray level of the } \\
\text { image so as to provide clues to the existence of a computed linear } \\
\text { structure }\end{array}$ \\
\hline
\end{tabular}

GLCM method testing assesses nonverbal communication carried out on the face, mouth, and eye frames that express Optimistic, Doubtful, Confused gestures. The emergence of the value of contrast, energy, entropy, homogeneity and correlation shows the consistency of one's expression (Figure 6a). The results

\begin{tabular}{|c|c|c|c|c|c|c|}
\hline \multicolumn{2}{|c|}{ Source Data } & Contras & Energy & Entropy & Homogenity & Corelasi \\
\hline \multirow{6}{*}{ Video $\mathrm{A}$} & Face 1 & 0,1295 & 0,1034 & 0,0531 & 0,382 & 0,473 \\
\hline & Face 2 & 0,1338 & 0,1092 & 0,073 & 0,835 & 0,643 \\
\hline & eyes 1 & 0,093 & 0,035 & 0,0872 & 0,924 & 0,563 \\
\hline & eyes 2 & 0,072 & 0,843 & 0,0832 & 0,734 & 0,842 \\
\hline & \begin{tabular}{|l|} 
Mouth 1 \\
\end{tabular} & 0,032 & 0,358 & 0,532 & 0,357 & 0,435 \\
\hline & Mouth 2 & 0,045 & 0,003 & 0,042 & 0,034 & 0,894 \\
\hline \multirow{6}{*}{ Video B } & Face 1 & 0,1053 & 0,1046 & 0,044 & 0,056 & 0,822 \\
\hline & Face 2 & 0,1324 & 0,1035 & 0.024 & 0,035 & 0,678 \\
\hline & eyes 1 & 0,094 & 0,059 & 0,032 & 0,075 & 0,854 \\
\hline & eyes 2 & 0,026 & 0,039 & 0,024 & 0,035 & 0,569 \\
\hline & Mouth 1 & 0,064 & 0,035 & 0,035 & 0,078 & 0,875 \\
\hline & Mouth 2 & 0,034 & 0,03 & 0,049 & 0,076 & 0,567 \\
\hline \multirow{6}{*}{ Video C } & Face 1 & 0,1335 & 0,1078 & 0,005 & 0,034 & 0,845 \\
\hline & Face 2 & 0,1825 & 0,1023 & 0,036 & 0,046 & 0,45 \\
\hline & eyes 1 & 0,035 & 0,092 & 0,083 & 0,023 & 0,569 \\
\hline & eyes 2 & 0,004 & 0,103 & 0,046 & 0,034 & 0,987 \\
\hline & Mouth 1 & 0,008 & 0,078 & 0,037 & 0,013 & 0,185 \\
\hline & Mouth 2 & 0,0342 & 0,024 & 0,062 & 0,048 & 0,556 \\
\hline
\end{tabular}

a. GLCM Formula Results

Figure 6 GLCM method implementation

\begin{tabular}{|c|c|c|c|c|c|c|}
\hline \multicolumn{2}{|c|}{ Source Data } & \multirow{2}{*}{\begin{tabular}{|r|} 
Angle 0 \\
0,565
\end{tabular}} & \multirow{2}{*}{\begin{tabular}{|r|} 
Angle 45 \\
0,585 \\
\end{tabular}} & \multirow{2}{*}{$\begin{array}{r}\text { Angle } 90 \\
0,78 \\
\end{array}$} & \multirow{2}{*}{\begin{tabular}{|r|} 
Angle 135 \\
0,784 \\
\end{tabular}} & \multirow{2}{*}{$\begin{array}{r}\text { Angle 180 } \\
0,45 \\
\end{array}$} \\
\hline \multirow{6}{*}{ A } & Face 1 & & & & & \\
\hline & Face 2 & 0,574 & 0,678 & \begin{tabular}{l|l|l|}
0,678 & \\
\end{tabular} & 0,7834 & 0.567 \\
\hline & eyes 1 & 0,7845 & 0,746 & \begin{tabular}{l|l}
0,86 \\
\end{tabular} & 0,3578 & 0,7078 \\
\hline & eyes 2 & 0,365 & 0,89 & 0,842 & 0,4534 & 0,6797 \\
\hline & Mouth 1 & 0,673 & 0,276 & \begin{tabular}{|l|l|}
0,945 & \\
\end{tabular} & 0,467 & 0,467 \\
\hline & Mouth 2 & 0,699 & 0,033 & 0,235 & 0,346 & 0,878 \\
\hline \multirow{6}{*}{ B } & Face 1 & 0,984 & 0,784 & \begin{tabular}{|l|l|}
0,673 \\
\end{tabular} & 0,335 & 0,987 \\
\hline & Face 2 & 0,564 & 0,789 & 0,3673 & 0,875 & 0,678 \\
\hline & eyes 1 & 0,745 & 0,959 & 0,3564 & 0,453 & 0,56789 \\
\hline & eyes 2 & 0,784 & 0,567 & \begin{tabular}{l|l|}
0,256 & \\
\end{tabular} & 0,355 & 0,478 \\
\hline & Mouth 1 & 0,567 & 0,562 & 0,4763 & 0,7834 & 0,74 \\
\hline & Mouth 2 & 0,795 & 0,904 & \begin{tabular}{|l|}
0,346 \\
\end{tabular} & 0,235 & 0,678 \\
\hline \multirow{6}{*}{ c } & Face 1 & 0,965 & 0,7856 & 0,764 & 0,346 & 0,7567 \\
\hline & Face 2 & 0,947 & 0,034 & \begin{tabular}{|l|l|}
0,367 \\
\end{tabular} & 0,574 & 0,679 \\
\hline & eyes 1 & 0,784 & 0,893 & \begin{tabular}{l|l}
0,346 \\
\end{tabular} & 0,789 & 0,456 \\
\hline & eyes 2 & 0,564 & 0,3566 & \begin{tabular}{|l|l|}
0,345 &
\end{tabular} & 0,988 & 0,345 \\
\hline & Mouth 1 & 0,675 & 0,7845 & 0,3307 & 0,789 & 0,889 \\
\hline & Mouth 2 & 0,53 & 0,567 & \begin{tabular}{|l|}
0,367 \\
\end{tabular} & 0,456 & 0,3458 \\
\hline
\end{tabular}

a. Fitur GLCM Angle of the application stage of the GLCM formula (Figure $6 b)$. The extraction process then applies the four different GLCM angle features, namely $0^{\circ}, 45^{\circ}, 90^{\circ}$, and $135^{\circ}$ (Figure 7a). Following are the results of the feature extraction of the GLCM angle (Figure 7b).

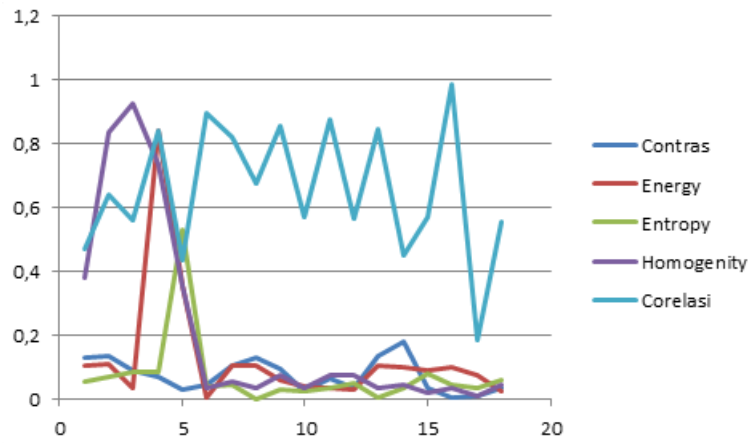

b. GLCM Value Appearance Graph

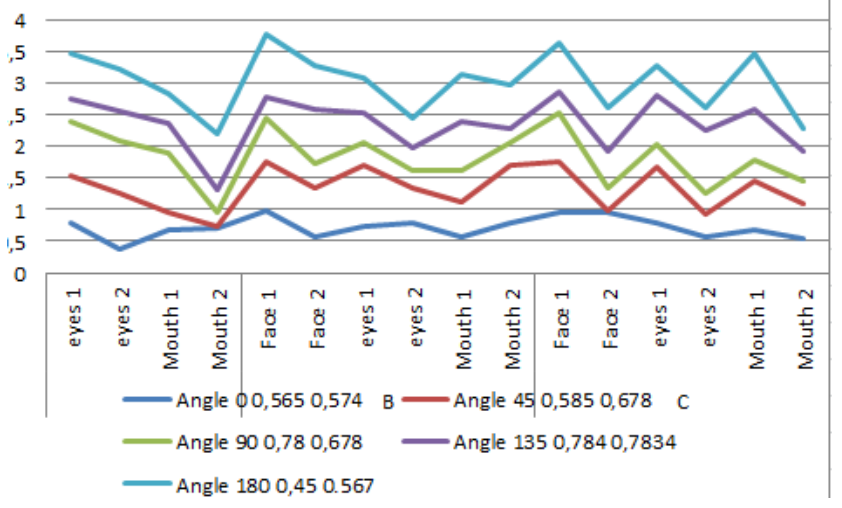

b. GLCM Angle Graph

Figure 7 GLCM angle result 
LBP assigns pixel labels to an image based on the neighboring thresholding of each pixel and represents it in binary form. LBP is proposed as a two-tiered version of the texture unit to describe local texture patterns. LBP values of images were taken to be classified to determine the facial expression of Optimist (Cluster 1), Confused (Cluster 2), Doubtful (Cluster 3). The data attributes consist of image size, threshold value, LBP value. The pixel value of the grayscale image is split into several $3 \times 3$ matrix regions, Figure 8.
From the clustering carried out, the results obtained are the objective function values during the iteration, the cluster center or center and the degree of graduate membership for each cluster in the last iteration. Repetition is based on minimizing the distance from the data point given to the cluster center on the degree of membership of the data point. The output of the Fuzzy CMeans algorithm is the degree of membership and the cluster center of each data point. After the iteration process, the cluster center grouping is obtained, Figure 9

\begin{tabular}{|c|c|c|c|c|c|c|c|c|}
\hline 177 & 178 & 178 & 1 & 1 & 1 & 177 & 178 & 178 \\
\hline 175 & 177 & 175 & 0 & & 0 & 175 & 13 & 175 \\
\hline 172 & 174 & 173 & 0 & 0 & 0 & 172 & 174 & 173 \\
\hline \multicolumn{3}{|c|}{ Matrix $3 \times 3$ Region 1} & \multicolumn{3}{|c|}{ Matrix Biner } & \multicolumn{3}{|c|}{ LBP } \\
\hline \multicolumn{9}{|c|}{ Region 1 , Binary $=1110000$ Desimal $=13$} \\
\hline 178 & 178 & 177 & 1 & 1 & 1 & 177 & 178 & 178 \\
\hline 177 & 175 & 174 & 1 & & 0 & 175 & 45 & 175 \\
\hline 174 & 173 & 169 & 0 & 0 & 0 & 172 & 174 & 173 \\
\hline \multicolumn{3}{|c|}{ Matrix $3 \times 3$ Region 2} & \multicolumn{3}{|c|}{ Matrix Biner } & \multicolumn{3}{|c|}{ LBP } \\
\hline
\end{tabular}

Figure 8 Change in region matrix using LBP extraction

Expression Cluster Center

\begin{tabular}{|l|l|l|}
\hline 5.8974 & 7.8243 & 6.9332 \\
\hline 5.7382 & 6.5392 & 8.3894 \\
\hline 5.9243 & 5.9243 & 7.1027 \\
\hline
\end{tabular}

Figure 9 FCM result graph

\subsection{Discussion}

Body language behavior is tracked into several frames according to the test data category. The assessment is made from the results of the video that has been recorded by analyzing the texture of the mouth, eyes and facial expressions. The Gray-Level Co-Occurrence Matrix (GLCM) method identifies statistical features in second-order form. Feature extraction was carried out based on parameters of contrast, correlation, energy, and homogeneity. Image geometry rotation operation to improve image quality and find the same pattern. The pixels at the $\mathrm{x}, \mathrm{y}$ coordinates represent the pixel neighbors that are tangent to each other.

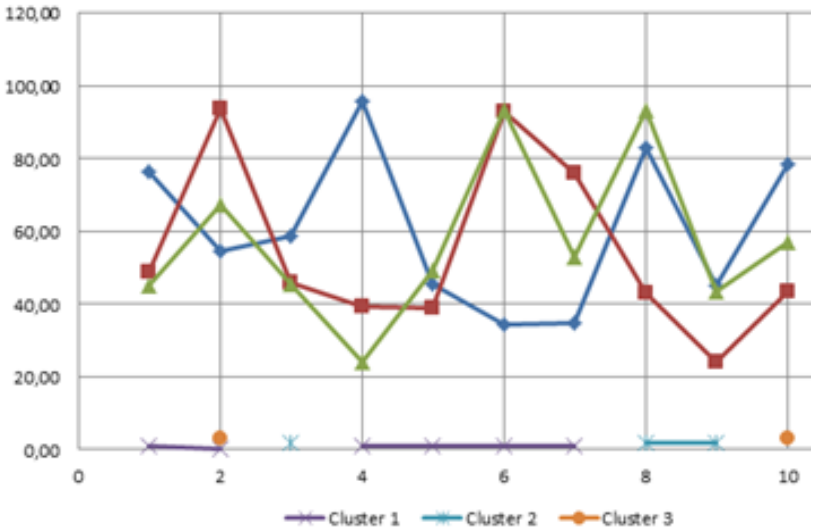

FCM clustering produces objective function values during the iteration, cluster center or center and graduate membership degrees for each cluster in the last iteration. Iteration is based on minimizing the distance from a given data point to the cluster center on the membership degree of that data point. The output of the Fuzzy CMeans algorithm is the degree of membership and the cluster center of each data point. After the iteration process, the cluster center grouping is obtained. Assessment of non-verbal communication movements was obtained from testing the appearance of facial, eye and mouth expression movements from the results of video tracking. 


\section{CONCLUSION}

The results showed that image identification using the Gray-Level Co-Occurrence Matrix (GLCM) method is influenced by the position of the object at the $x, y$ coordinates, the distance of pixels, the intensity values of black and white images ( 0 and 1$)$ to form the characteristics of the assessment extract based on the average appearance of the pattern. the same from the video tracking results. The feature extraction pattern requires a repeating process of patterns, intensity and statistical distribution to obtain certain characteristics of the image. Clustering with Fuzzy C-Means (FCM) informs the grouping of membership degrees for each data based on the occurrence of 0 and 1 from the image extract.

\section{ACKNOWLEDGMENTS}

Thank you so much for Ristek Brin and Kemendikbud for research Grant 2019.

\section{REFERENCES}

[1] X. Huang, X. Liu, and L. Zhang, "A multichannel gray level co-occurrence matrix for multi/hyperspectral image texture representation," Remote Sens., vol. 6, no. 9, pp. 8424-8445, 2014, doi: $10.3390 / \mathrm{rs} 6098424$.

[2] A. S. R. M. Sinaga and A. S. Sitio, "Real Time Catalog of Uniqueness Face Using the CAMShift and Gabor Wavelet Algorithims," J. Phys. Conf. Ser., vol. 1424, no. 1, 2019, doi: 10.1088/17426596/1424/1/012012.

[3] M. Yusuf, A. Muntasa, W. Agustiono, D. R. Anamisa, and M. Syarief, "A Novel Conceptual Model of e-Participation using Biometrics Technologies," J. Phys. Conf. Ser., vol. 1569, no. 2, 2020, doi: 10.1088/1742-6596/1569/2/022069.

[4] W. Y. Leng, S. M. Shamsuddin, and S. Sulaiman, "Enhancement of Individuality Representation for
Multi-Biometric Identification," IOP Conf. Ser. Mater. Sci. Eng., vol. 884, no. 1, 2020, doi: 10.1088/1757-899X/884/1/012061.

[5] I. U. W. Mulyono et al., "Parijoto Fruits Classification using K-Nearest Neighbor Based on Gray Level Co-Occurrence Matrix Texture Extraction," J. Phys. Conf. Ser., vol. 1501, no. 1, 2020, doi: 10.1088/1742-6596/1501/1/012017.

[6] W. Han, H. Zhao, Z. Zhao, F. Gu, and D. Zhen, "Application of time-frequency image feature extraction method based on gray level cooccurrence matrix in coal mine pipeline leakage detection," IOP Conf. Ser. Earth Environ. Sci., vol. 446, no. 5, 2020, doi: 10.1088/1755$1315 / 446 / 5 / 052054$.

[7] M. Z. Osman, M. A. Maarof, and M. F. Rohani, "Texture-based Feature using Multi-blocks Gray Level Co-occurrence Matrix for Ethnicity Identification," IOP Conf. Ser. Mater. Sci. Eng., vol. 769, no. 1, 2020, doi: 10.1088/1757$899 X / 769 / 1 / 012032$.

[8] Y. Hendrawan et al., "Development of colour cooccurrence matrix (CCM) texture analysis for biosensing," IOP Conf. Ser. Earth Environ. Sci., vol. 230, no. 1, 2019, doi: 10.1088/1755$1315 / 230 / 1 / 012022$.

[9] R. A. Asmara, R. D. R. Yusron, F. Rahutomo, R. Ariyanto, D. K. P. Aji, and P. Choirina, "Iris feature extraction using Gray Level Co-occurrence Matrix and Gabor Kernel filter its impact on iris Huffman compression image," J. Phys. Conf. Ser., vol. 1402, no. 6, 2019.

[10] Z. Ji, S. Wu, F. Wang, L. Xu, Y. Yang, and X. Hu, "Mining Regional Co-Occurrence Patterns for Image Classification," Math. Probl. Eng., vol. 2018, 2018. 